Hydrol. Earth Syst. Sci., 10, 197-208, 2006

www.hydrol-earth-syst-sci.net/10/197/2006/

(C) Author(s) 2006. This work is licensed

under a Creative Commons License.

\title{
Distance in spatial interpolation of daily rain gauge data
}

\author{
B. Ahrens ${ }^{1, *}$ \\ ${ }^{1}$ Institut für Meteorologie und Geophysik, Universität Wien, Austria \\ *now at: Institute for Atmosphere and Climate, ETH Zurich, Switzerland
}

Received: 10 August 2005 - Published in Hydrol. Earth Syst. Sci. Discuss.: 8 September 2005

Revised: 29 November 2005 - Accepted: 7 February 2006 - Published: 4 April 2006

\begin{abstract}
Spatial interpolation of rain gauge data is important in forcing of hydrological simulations or evaluation of weather predictions, for example. This paper investigates the application of statistical distance, like one minus common variance of observation time series, between data sites instead of geographical distance in interpolation. Here, as a typical representative of interpolation methods the inverse distance weighting interpolation is applied and the test data is daily precipitation observed in Austria. Choosing statistical distance instead of geographical distance in interpolation of available coarse network observations to sites of a denser network, which is not reporting for the interpolation date, yields more robust interpolation results. The most distinct performance enhancement is in or close to mountainous terrain. Therefore, application of statistical distance in the inverse distance weighting interpolation or in similar methods can parsimoniously densify the currently available observation network. Additionally, the success further motivates search for conceptual rain-orography interaction models as components of spatial rain interpolation algorithms in mountainous terrain.
\end{abstract}

\section{Introduction}

Precipitation maps with daily or better resolution are necessary for investigation of the climatology of extreme events (e.g. Skoda et al., 2003; Palecki et al., 2005), as input in hydrological modeling (e.g. Singh and Frevert, 2002a,b), or in evaluation of numerical weather prediction models (e.g. Beck et al., 2004), for example. Depending on application the maps have to be available close to real-time (e.g. in detection of flood generating processes) or it is possible to wait

Correspondence to: B. Ahrens

(bodo.ahrens@env.ethz.ch) some time and gather as much rain observation data as possible (e.g. in climatology).

The back-bone of these maps are rain gauge data since the reliability of remote sensing data (e.g., by weather radar or satellite) is not high enough (e.g., Young et al., 1999; Ciach et al., 2000; Adler et al., 2001). A challenge in mapping is the temporal variation of spatial coverage of available rain gauges. For example, the monthly monitoring product of the Global Precipitation Climatology Centre (http://gpcc.dwd.de) is based on about 6000 stations available in near real-time. A second product, the so-called full product, is based on 40000 stations in the late 1980s but based on only about 20000 stations in the year 2000 . Another example of time-delay in data availability is a daily precipitation atlas by Rubel (1996) with gridspacing of a few tens of kilometers for the Baltic sea and its drainage basin (area: $1.7 \mathrm{e} 6 \mathrm{~km}^{2}$ ). Rubel (1996) is based on about 400 stations and its update by Rubel and Hantel (2001) is based on a 10-times denser station network. Liebmann and Allured (2005) gives a very recent example of varying observation network density in precipitation mapping.

The essence of precipitation mapping is the interpolation of point data (the rain gauge orifices of $\sim 1000 \mathrm{~cm}^{2}$ are small compared to the mapping scale, thus the observation sites are considered to be points in good approximation) and spatial averaging or smoothing of the interpolated point data. This leads to precipitation fields with spatial gridspacing and cell support of, for example, a few tens of kilometers. Mapping of rain gauge data is a point-to-area interpolation of the available information.

Auer et al. (2005) developed a homogenized data set of long series of monthly precipitation at 192 station sites in the European Alps and their surroundings. Relative series homogenization relies on significant common variability between neighbored site series assumed to be expressible as common variance $R^{2}$ with $R$ the linear correlation coefficient. Auer et al. (2005) chose a threshold of $R^{2}=0.5$. But,

Published by Copernicus GmbH on behalf of the European Geosciences Union. 


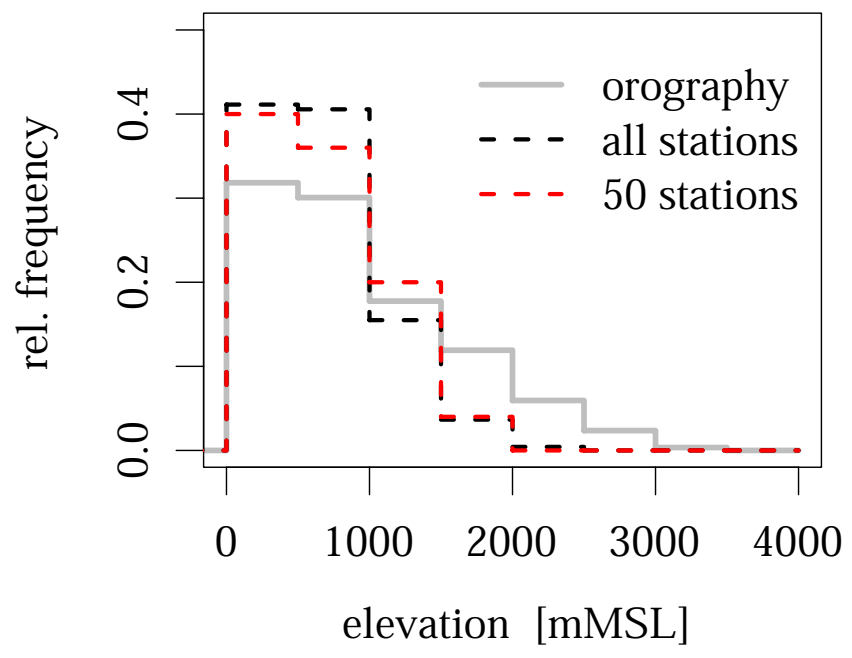

Fig. 1. Height distributions of the Austrian orography, of all considered rain gauges, and of a subset of 50 gauges on an approximately regular spatial grid.

Scheifinger et al. (2003) estimated that on average a network density of about $1 / 100 \mathrm{~km}$ is necessary for establishing $R^{2} \geq 0.5$ in the greater Alpine region, and the average siteto-site distance increases backwards in time from $61 \mathrm{~km}$ in the second half of the 20th century to $74 \mathrm{~km}$ in the late 19th century and up to about $200 \mathrm{~km}$ in the early 19th century. Thus relative homogenization is not possible in the early 19 th century. Relative series homogenization is a point-to-point transformation or interpolation of information.

Another, closely related point-to-point interpolation challenge is estimation of missing data, i.e. filling in the precipitation time series of a temporarily not reporting station by using information of neighboring stations. Methods for filling in are, for example, inverse distance weighting (IDW) interpolation, ordinary Kriging, or multiple linear regression using the least absolute deviations criterium (Eischeid et al., 2000). These authors concluded that in interpolation of daily precipitation data "the preselection of surrounding stations, based on their relationship with the station to be estimated, is an integral first step" of all interpolation methods, but with least absolute regression outperforming the other methods in their application.

The relationship between stations is considered differently in the mentioned interpolation methods. The standard IDW method expresses relationship in terms of geographical distance; the Kriging variants apply variogram models which are in typical implementations monotonic functions of geographical distance; and multiple regression applies some statistical distance between the observation time series. Therefore, Tobler's first law of geography (Tobler, 1970) that all things are related, but nearby things are more related than distant things, is respected in all methods, but with different interpretations of distance.
This paper discusses application of different statistical distances instead of geographical distance in interpolation of observations of a coarse station network to station sites that are not reporting at the interpolation date. Therefore, it discusses the filling in challenge. But, here the challenge is filling in hundreds of observations of a fine-grid station network from an available coarse-grid network with varying network density. The data sets, here from Austria, will be introduced in the next section. The goal is to test a parsimonious method for effective network densification that has the potential to improve rainfall mapping. Section 3 explains how to use some statistical distance measure instead of geographical distance in the often applied, easily to comprehend and implement IDW method. Section 3 explains why IDW is an ideal vehicle for illustrating the advantages and disadvantages of statistical distance and interpolation results will be shown in Sect. 4. Finally, some conclusions and a brief outlook are given.

\section{Data}

For evaluation of precipitation interpolation methods assuming different mean observing station distances a dense reference network of precipitation stations is necessary. In this investigation a data set of about 900 stations with long daily time series (in the period 1971 to 2002) has been available for Austria (total area is $84000 \mathrm{~km}^{2}$ ) as provided by the Hydrographisches Zentralbüro, Vienna (delivery date: February 2005). Austria is a country with $62 \%$ covered by the Austrian Alps and only $32 \%$ below $500 \mathrm{~m}$, cf. Fig. 1, and thus interpolation is done in complex mountainous terrain.

The chosen year for the interpolation experiments is 1999 . All stations with missing data in 1999 and not at least twenty years of data are erased from the data set and the investigations are done with the remaining 710 station time series. This set of stations is named ALL in the following.

In this set of ALL stations the mean next station interdistance is $6.7 \mathrm{~km}$, but the stations are not regularly distributed within the domain of investigation. They are clustered around Vienna in the north-east of the domain and in the main Alpine valley floors (Fig. 2). The irregularity is also illustrated in Fig. 1 which compares the orographic height distribution with distributions of station heights. The lower altitudes are relatively better represented by stations than higher elevations.

In the interpolation experiments this paper applies subsets of ALL stations with 25, 50, and 150 members as observing stations and subsets of the remaining stations with $300 \mathrm{mem}-$ bers as evaluating stations, which are considered in the interpolation experiments as temporarily not reporting station but with a long time series of data. The subsets are drawn in a fashion that approximately maximizes the next station geographic inter-distance. One station of the minimum distance pair is erased until the wished number of observing and 


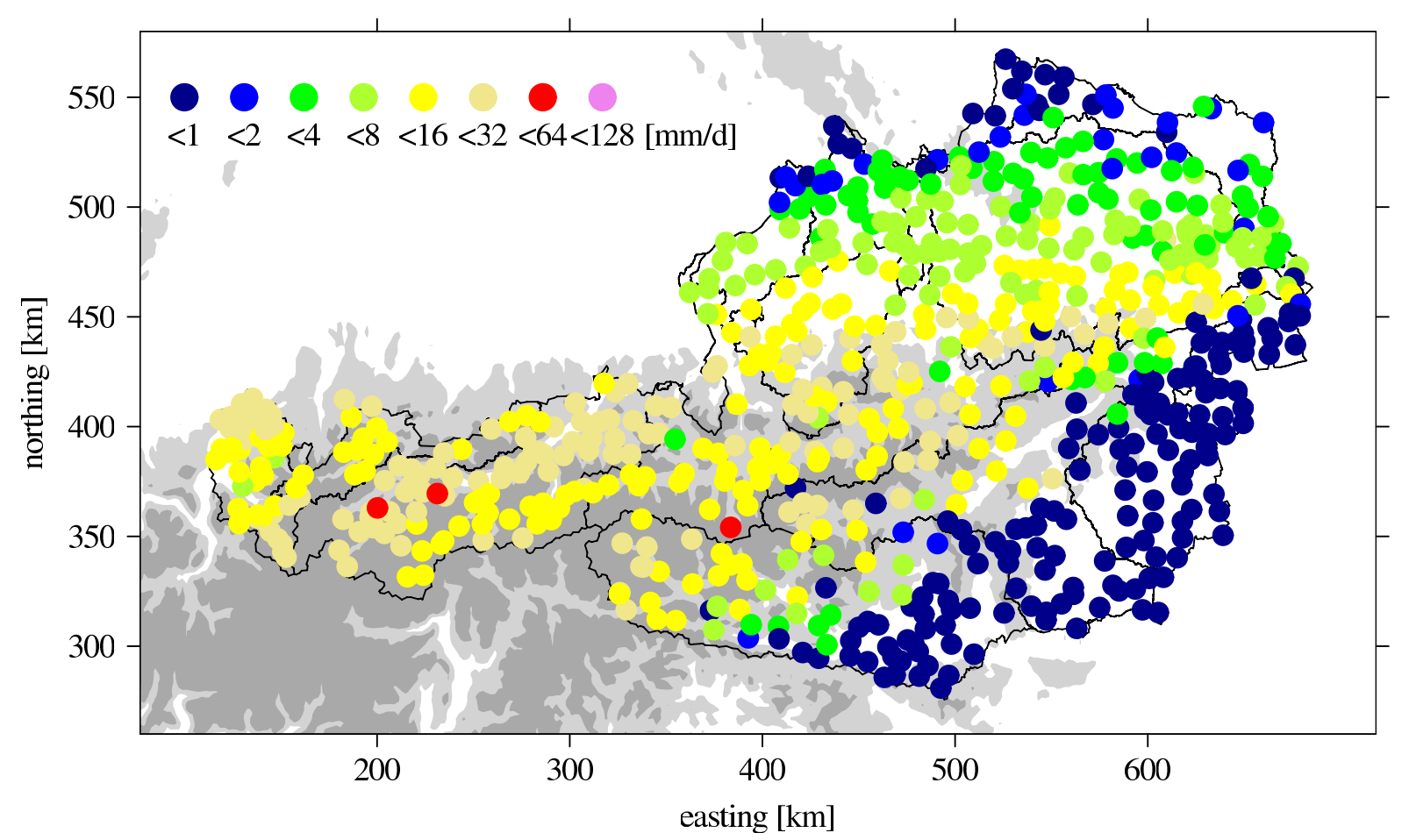

Fig. 2. Rain gauges locations of set ALL (bullets) considered in the present investigation and measured values (bullet colors) for 19 August 1999. The orography is indicated by grey shading (light-grey: elevations above $800 \mathrm{~m}$ m.s.l., and dark-grey: elevations above $1500 \mathrm{~m}$ m.s.l.). The main Austrian watersheds are indicated by black isolines.

subsequently of evaluating stations is left. Table 1 gives minimum, mean, and maximum geographic distance and next neighbor common variance $R^{2}$. The mean $R^{2}$ increases with number of stations as expected and consequently the mean interpolation performance should increase. It is noteworthy that for all station sets there are sites which are statistically far from all the others, i.e. $R^{2}<0.5$. The number of 150 stations is chosen since this is about the number of stations that are operational at the Austrian national weather service. This is a globally comparably dense operational network. But, the data of the weather service is not used here since different data sets with different measurement device types and quality control shall be avoided here for the sake of simplicity.

The chosen regularizing sub-sampling leads to declustering of the considered station sets, but as Fig. 1 illustrates the elevation distribution of the stations is only slightly improved. It should be kept in mind that typical station networks are clustered and thus the effective number of stations in mapping is smaller than the nominal number of stations. Random sub-sampling experiments lead to decreasing interpolation performance, but this will not be discussed further.

In climatological mapping of precipitation in mountainous terrain a precipitation-elevation relationship is often successfully considered (cf. Sevruk, 1997). This elevation dependence is illustrated in Fig. 3 for yearly data. It is also illustrated that such a dependence is less obvious at shorter time scales because of the large scatter of the daily precipitation
Table 1. Statistics of the geographic distances of the considered station sets and statistics of the common variance $R^{2}$ of daily precipitation series.

\begin{tabular}{lcccccc}
\hline \multirow{2}{*}{ set } & \multicolumn{3}{c}{ distance $[\mathrm{km}]$} & \multicolumn{3}{c}{$R^{2}[\%]$} \\
& $\min$ & mean & $\max$ & $\min$ & $\operatorname{mean}$ & $\max$ \\
\hline ALL/710 & 1 & 7 & 21 & 40 & 73 & 97 \\
25 & 43 & 54 & 69 & 28 & 41 & 53 \\
50 & 29 & 36 & 53 & 29 & 50 & 69 \\
150 & 16 & 20 & 37 & 41 & 61 & 77 \\
\hline
\end{tabular}

values. This will be discussed in some more detail in the following section.

\section{Interpolation method}

For illustrational purposes the Inverse Distance Weighting interpolation (IDW) method is applied. IDW assigns weights to neighboring observed values based on distance to the interpolation location and the interpolated value is the weighted average of the observations. IDW is applied in many precipitation mapping methods (e.g., Rudolf and Rubel, 2005; Frei and Schär, 1998) often enhanced with add-ons like declustering and directional grouping of stations, or empirical 


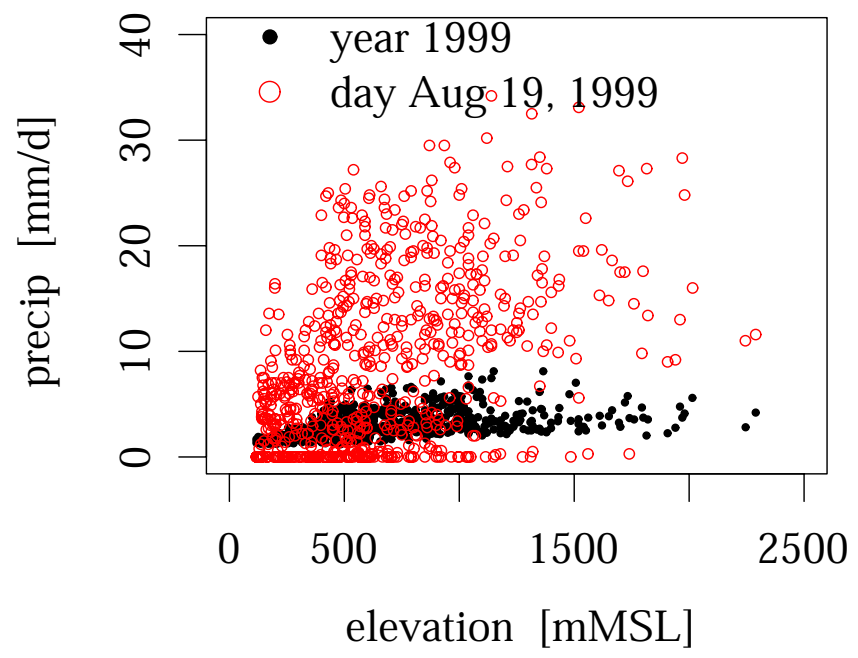

Fig. 3. Height dependence of precipitation observed by all stations for the year 1999 and the day 19 August 1999.

adjustments in respect to orography (Daly et al., 1994). The IDW method is a simple, but efficient interpolation method. It is shown that statistical interpolation methods like multiple linear regression, optimal interpolation or Kriging can perform better, but only if data density is sufficient (Eischeid et al., 2000). Successful applications of Kriging and optimal interpolation are presented in Rubel and Hantel (2001) and Durand et al. (1993), respectively.

Here, IDW is applied as an ideal vehicle to illustrate the effects of distance interpretation in interpolation. Standard IDW applies a geographical distance measure and this will be replaced with some statistical distance measure between observed time series at station sites. This is the main idea of the above mentioned statistical methods and will further be discussed below after the IDW has been introduced formally.

In standard IDW the interpolated value is estimated by a weighted mean of the observations and the weights are proportional to a negative power of geographical distances $d_{\alpha}$ between the point of interpolation and the considered observation points. Typically, not all observations $P_{\alpha}$ are considered in estimation of the interpolating value $P_{0}$ but only $n$ neighboring with

$P_{0}=\frac{\sum_{\alpha=1}^{n} P_{\alpha} w_{\alpha}}{\sum_{\alpha=1}^{n} w_{\alpha}}$

and the weights

$w_{\alpha}=1 / d_{\alpha}^{\lambda}$

The power $\lambda$ of distance has to be chosen appropriately depending on the interpolated variable. Spatially smoother variables show larger spatial dependence and thus like smaller values of $\lambda$ than spatially rougher fields. Generally, it is assumed that the separation of close-by observations increases faster than linear with station distance and often a power $\lambda$ of two is assumed.
If only the next neighbor is considered (i.e. $n=1$ ) IDW collapses to the next neighbor or Thiessen method. As Blöschl and Grayson (2001) elaborated, IDW generates spurious artefacts in case of highly variable quantities and irregularly spaced data sites. This is typical for observed precipitation data. Thus, in practical implementations the IDW is complemented by empirical methods like directional grouping of stations and exclusion of stations if shadowed by closer stations (Shepard, 1984). These artefacts are not important in our experiments because of the applied regularizing subsampling of the available stations. IDW interpolation applying geographical distance is named $d$-IDW in the following.

Besides geographical distance additional empirical relationships can be implemented. One example is regression with orography (Daly et al., 1994). Adopting this regression is crucial in development of climatological precipitation maps but of less importance in daily maps as Fig. 3 indicates. But this example illustrates that besides horizontal distance also vertical distance, slope of orography, observation positioning at the wind- or leeward slope, distance from the range crests etc. should be considered (Smith, 1979). Unfortunately, implementations of adequate empirical relations of that type are difficult (Smith, 2003; Barros and Lettenmaier, 1994). A simple station separation measure is wanted which takes the complexity of rain-terrain interaction into account.

Here, it is assumed that long time series of precipitation are available at the observation sites and the interpolation sites. Thus, it is easy to replace geographical distance by some type of statistical distance between data series. A proper statistical station distance implicitly considers rain-terrain interaction through experience. One useful class of statistical measures obviously are cross-correlation type measures like $1-R^{2}$. The drawback of this measure of proximity is that differences in the mean between neighboring series are not considered. Therefore, the semi-mean squared difference (i.e., basically the Euclidean distance)

$\gamma_{0 \alpha}=\frac{1}{2 T} \sum_{t=1}^{T}\left(P_{t 0}-P_{t \alpha}\right)^{2}$

between the time series of length $T$ is applied as an alternative statistical measure of separation. Only days $t$ with precipitation at both observation sites 0 and $\alpha$ are considered. This measure quantifies random and systematic differences between the time series.

If, instead of $d$, the $\gamma$ is applied in IDW the proximity of stations is replaced with the proximity of data series. The resulting interpolation method is named $\gamma$-IDW in the following.

Application of $d$ - or $\gamma$-IDW yields different interpolation values since geographical close-by stations can observe relatively distant precipitation time series and vice versa. This is shown in Fig. 4. For single evaluating stations the next geographical neighbor might not be the next neighbor measured by the $\gamma$-distance (exemplified by two station's $\gamma$-vectors 


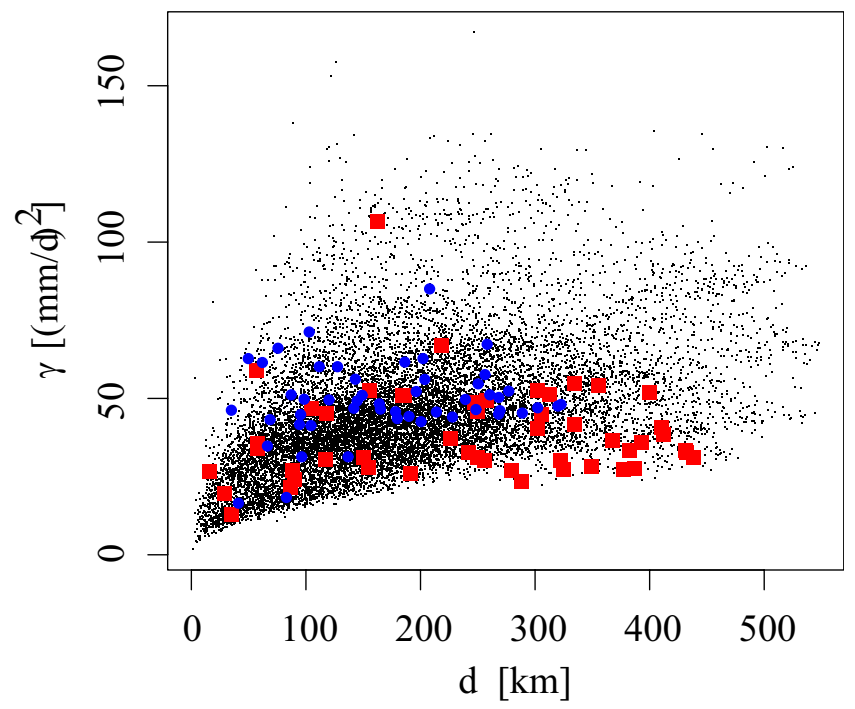

Fig. 4. Statistical distance $\gamma$ for all evaluation-observation pairs. Here, 50 observing stations are assumed. The $\gamma$ s for two stations (cf. the stations marked by colored arrows in Fig. 7) are highlighted by colored symbols.

marked red and blue in the figure). Therefore, application of the $\gamma \mathrm{s}$ instead of the $d \mathrm{~s}$ changes the selection of the $n$ next neighbors and their relative importance in the interpolated value.

The factor $1 / 2$ in the definition of $\gamma$ is not important here, but chosen to illuminate that the scattergram shown in Fig. 4 would be the empirical semi-variogram in case of Kriging with a climatological semi-variogram like in Rubel and Hantel (2001). In case of a stationary field the Kriging method applying a climatological semi-variogram is equivalent to Gandin's optimal interpolation where distance is measured in terms of time-series correlation, and both are very similar to the classical multiple linear regression (Creutin and Obled, 1982). In multiple linear regression the correlations between interpolation and observation sites are known. Kriging and optimal interpolation are applied in mapping where these correlations are generally unknown and have to be replaced by variogram or correlation models, respectively.

The advantage of these methods over statistical distance IDW is that they account for relationships between observing stations. Therefore, statistical IDW can be considered as a simplified prototype of these more elaborated interpolation methods. The IDW is an easy framework for investigating the impact of replacing geographical distance with some statistical distance between observation and interpolation sites. Algorithmically the change in distance interpretation is easily done by replacing the geographical distance with a statistical distance matrix. This is also an advantage if operational application is considered since IDW variants are often applied in mapping schemes and since, for example, in multiple linear regression the regression coefficients have to

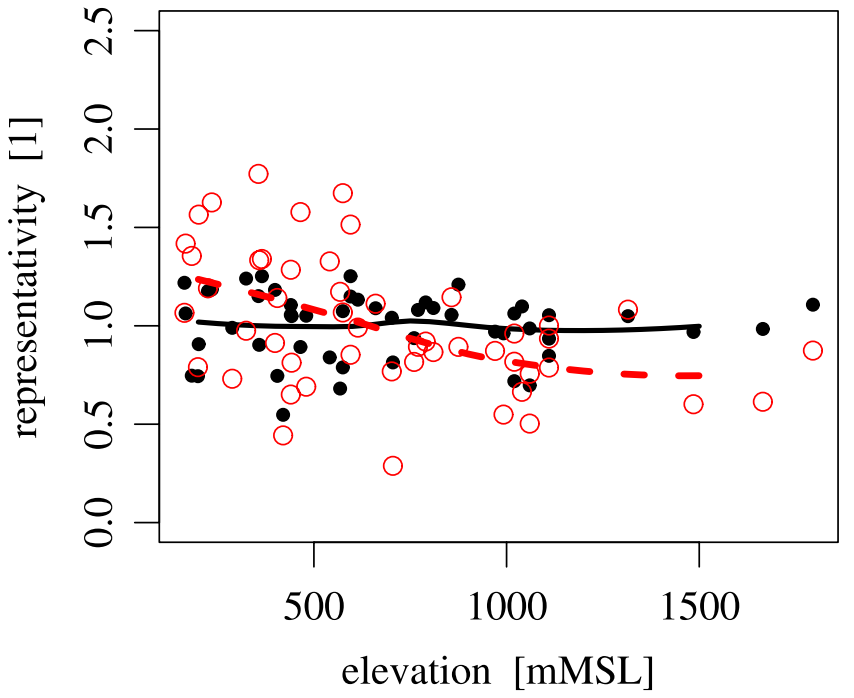

Fig. 5. Relative representativeness in geographical (black bullets) and $\gamma$-statistical (red circles) of the observing stations of set 50 . The solid black and dashed red lines are local polynomial regression fits to the bullets and circles, respectively.

be estimated and algorithmically dealt with for each interpolation site and network topography separately, and this is a formidable task.

Figure 5 indicates the spatial and statistical representativeness of the observing stations of set 50. Shown are the averaged inverse distances to the neighboring 24 evaluating station sites (i.e. about to neighbored evaluating sites to which the observing stations are applied to in interpolation with $n=4)$. The geographical representativeness of the stations scatter but is not systematically dependent on station elevation. This confirms that the regularizing sub-setting has been successful. The statistical representativeness decreases with station elevation on average. Since this is not respected by geographical distance weighting and since mean observed precipitation increases with height it is expected that precipitation will be tendencially overestimated in the Alpine area by interpolation with $d$-IDW. Additionally, $n$ might be chosen larger or the exponents $\lambda$ chosen smaller in the eastern lowlands of Austria than in the Alpine area in an optimized $d$-IDW interpolation setup to compensate the varying data representativeness (not tested here).

As mentioned above the $\gamma$ s also measure systematic differences in time series which may be due to elevation dependence of precipitation in orography, mountain shadowing effects, or horizontal trends in the precipitation field, for example. These systematic differences are not measured by the centered semi-mean squared difference

$\gamma_{0 \alpha}^{\prime}=\frac{1}{2 T} \sum_{t=1}^{T}\left(\left(P_{t 0}-m_{0}\right)-\left(P_{t \alpha}-m_{\alpha}\right)\right)^{2}$

with $m$ the time series means. 


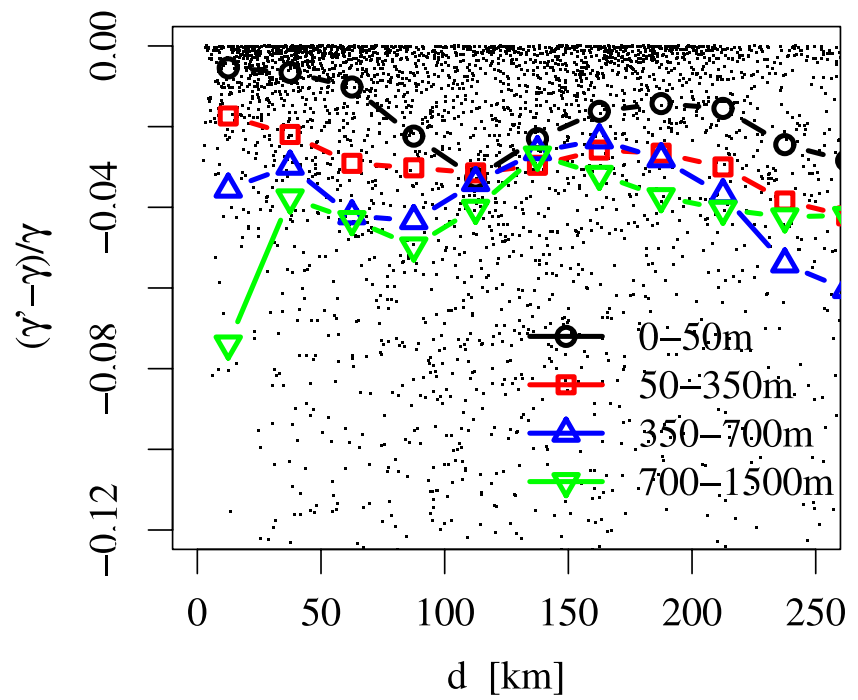

Fig. 6. Relative importance of systematic differences between pairs of precipitation time series over geographical distance between the series sites. The black dots show the relative importance for station pairs with a vertical elevation difference of less than $50 \mathrm{~m}$ (only every 5 th dot is drawn). The black circles show mean relative importances for geographical distance classes. The same is shown by the other colored symbols but for different vertical elevation difference classes.

The effects of systematic differences on statistical distances are shown in Fig. 6. The relative effect $\left(\gamma^{\prime}-\gamma\right) / \gamma$ over geographical distance is given for four classes of station elevation differences. For geographically nearby stations the importance of systematic differences is increasing with station elevation difference. On average the effect of systematic difference between nearby stations with almost no vertical elevation difference is below $1 \%$. This effect is about $7 \%$ for stations with about $1000 \mathrm{~m}$ vertical distance. This is consistent with Haiden and Stadlbacher (2002). They found for the same data an elevation dependence of yearly precipitation amounts up to $20 \%$ per $1000 \mathrm{~m}$ height difference if they restricted their evaluation to station pairs with horizontal distances smaller than ten kilometers.

With increasing horizontal distance the height difference gets less important. For $d \leq 100 \mathrm{~km}$ trends due to shadowing effects in complex terrain might be important and the remaining height correlation shown in Fig. 6 might be due to an increasing shadowing probability with larger station height differences. For even larger horizontal distances a pronounced east-west gradient in Austrian precipitation sums (cf. Fig. 8) might explain the increasing differences between $\gamma$ and $\gamma^{\prime}$. The vertical difference dependence is probably artificial since the relative frequency of orographic heights differs substantially between eastern and western Austria. Here, the possible reasons of systematic effects are not further discussed, but the existence of systematic effects motivates the usage of $\gamma$ instead of $\gamma^{\prime}$ or $R^{2}$ as the statistical distance mea- sure. In either interpolation method these trends have to be considered adequately.

The $\gamma$-IDW can be applied only at interpolation sites with long time series of precipitation observations. At nonobservation sites a mixed method could be thought of. The $n$ next neighbors are determined by geographical distance. For the neighbors long precipitation time series are available and thus their $d$ and $\gamma$ inter-distances can be determined. With this information a simple approximation for statistical distances of the interpolation site to the next neighbors can be derived. Geometrical selection combined with approximated statistical distances and thus approximated statistical weights generates an interpolation method that is slightly better than $d$-IDW but shall not be further discussed here. The performance gain is small indicating that orogenic modifications on statistical distance are non-homogeneous and anisotropic in space.

\section{Results}

As already noted the interpolation experiments are done with the observing station data sets of size 25, 50, and 150 for the year 1999. Always 300 evaluating station sites are the considered interpolation points and thus evaluating data is available. Figure 7 compares the results of $d$ - and $\gamma$-IDW interpolation. In this example the number of observing stations is 50 and next-neighbor interpolation, i.e. $n=1$, is applied. It is shown that often the next neighbors and thus the interpolation values differ between the two approaches. In nextneighbor $\gamma$-IDW interpolation even two stations (Mitterfeldalm (1665 m m.s.1.) and Filzmoos (1060 m m.s.l.) circled in Fig. 7) are not considered in interpolation. The spatial representativeness of these stations is relatively small and thus the observations at these stations are statistically useless for next-neighbor interpolation.

The interpolation results are compared to the evaluating observations with simple statistics like relative bias $B=($ mean $(I)-$ mean $(O)) /$ mean $(O)$ with $I$ a set of interpolated values and $O$ the corresponding set of evaluating observations at the interpolation sites, linear correlation $R(I, O)$, the ratio of standard deviations $\sigma(I) / \sigma(O)$, and efficiency $E=1-\operatorname{mean}\left((I-O)^{2}\right) / \sigma^{2}(O)=1-\operatorname{MSE}(I, O) / \sigma^{2}(O)$.

The spatial average of time series biases is denoted by $B_{t}$. and the temporal average of biases between daily precipitation fields is denoted $B_{. s}$. The dot indicates the finally averaged dimension. The same notation is applied to the averaged correlations, standard deviations and efficiencies. In case of perfect interpolation the values of the bias statistics are identical zero and the other statistics' values are one.

Table 2 shows mean results of the evaluation. As expected the correlation of interpolated values with evaluating data increases with the number of observing stations. Improvement of bias is not that obvious. Changes in the exponent $\lambda$ have a smaller impact, but are not unimportant. The evaluation 


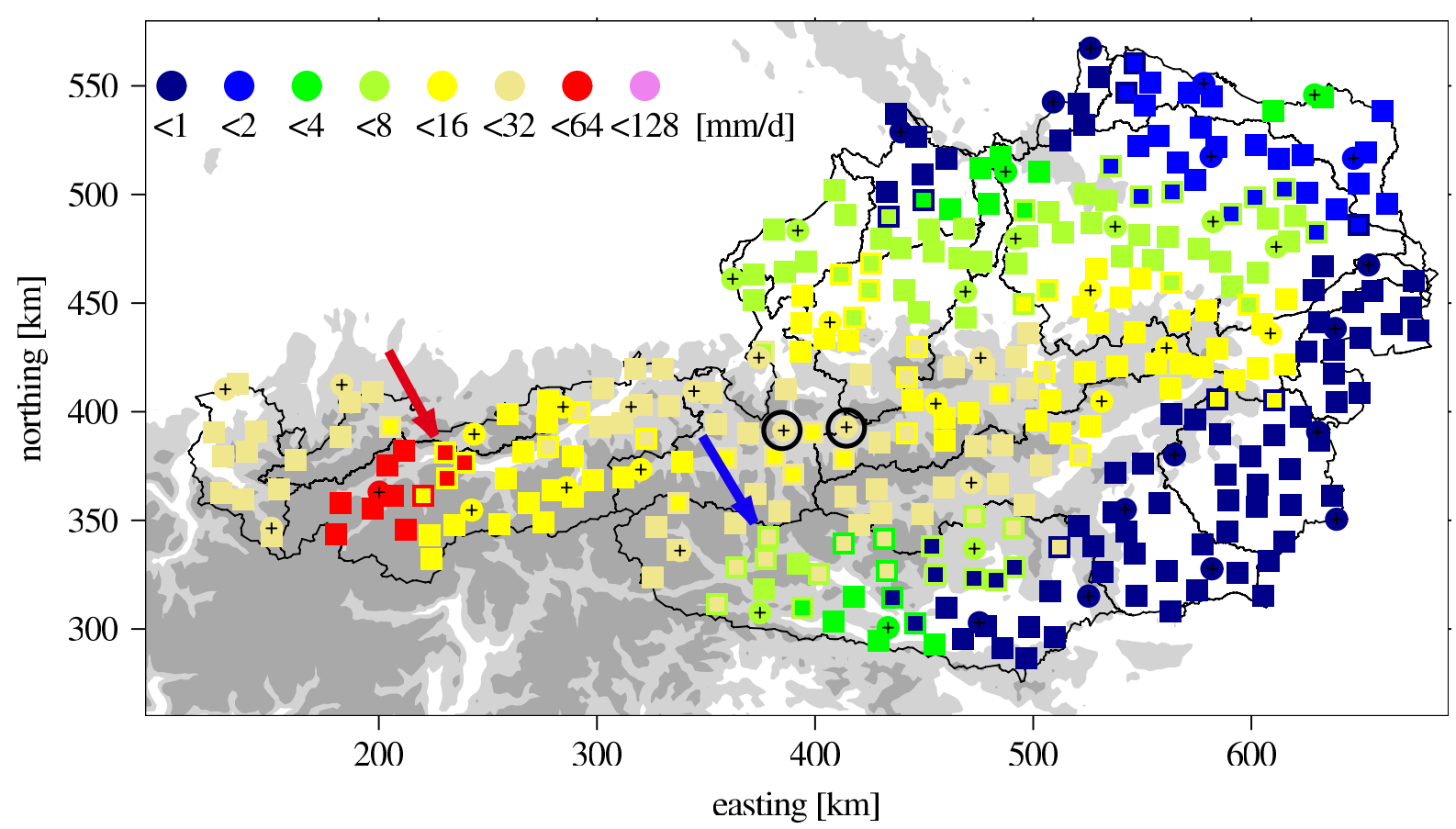

Fig. 7. IDW interpolation of data measured at 50 observing stations for 19 August 1999. The color of the bullets at the station sites (marked with +) show the observed values. These are a subset of the observations shown in Fig. 2. The squares indicate the interpolation points with the outline color giving the interpolation results by standard next-neighbor geographical IDW and the filling color giving next-neighbor $\gamma$-IDW results. The arrows point to the interpolation stations highlighted in Fig. 4. The circles mark observing stations not considered in next-neighbor $\gamma$-interpolation.

indicates that in $d$-IDW an exponent smaller than two performs best in the yearly average. More important is the number $n$ of considered observation neighbors. In case of 50 observing stations four-neighbor interpolation is better than next-neighbor interpolation but there is no relevant improvement in taking eight neighbors and even slight decrease in interpolation performance if sixteen neighbors are taken. A similar number of neighbors are optimal in case of 25 or 150 observing stations.

The $\gamma$-IDW interpolation is better in correlation and efficiency but seems to be worse in bias. In $d$-interpolation there is a more pronounced spatial compensation of errors. Underestimation, for example close to the southern Austrian border (cf. Fig. 8), is compensated by a tendency for overestimation in central Alpine areas by geographical interpolation. This is due to overestimation of representativeness of high-elevation observations (cf. Fig. 5). The tendency for underestimation in $\gamma$-interpolation can be avoided if the statistical distances are estimated after logarithmic transformation of the time series (cf. experiment $\mathrm{ln}$ in Table 2). This effectively reduces the positive skewness of the intensity distribution of daily precipitation. The skewness of the precipitation distribution is an important problem common to all interpolation methods, but shall not be discussed further in the present context.

Figure 8 shows the relative biases of the interpolating time series with next-neighbor $d$ - or $\gamma$-distance interpolation. The
Table 2. Mean evaluation results of interpolation experiments. The table gives the spatial mean of relative time series biases $\mathrm{B}_{t}$, the temporal mean of spatial biases is $\mathrm{B}_{. s}$, and the related correlation coefficients $R_{t}$. and $R_{. s}$ and efficiencies. All values are given in percent and thus the values of $B$ s would be 0 and all other values would be 100 in case of perfect interpolation.

\begin{tabular}{crrrrrr}
\hline $\begin{array}{c}\text { Exp. } \\
\text { set, } \lambda, n,-\end{array}$ & $\begin{array}{r}B_{t .} \\
d / \gamma\end{array}$ & $\begin{array}{r}B_{. s} \\
d / \gamma\end{array}$ & $\begin{array}{r}R_{t .} \\
d / \gamma\end{array}$ & $\begin{array}{r}R_{. s} \\
d / \gamma\end{array}$ & $\begin{array}{r}E_{t .} \\
d / \gamma\end{array}$ & $\begin{array}{r}E_{. s} \\
d / \gamma\end{array}$ \\
\hline $25,2,4,-$ & $1 /-5$ & $0 /-7$ & $84 / 85$ & $56 / 60$ & $67 / 71$ & $22 / 33$ \\
$50,2,4,-$ & $4 /-3$ & $1 /-7$ & $87 / 88$ & $62 / 65$ & $73 / 76$ & $32 / 42$ \\
$150,2,4,-$ & $1 /-2$ & $-1 /-5$ & $91 / 91$ & $70 / 72$ & $80 / 82$ & $45 / 52$ \\
& & & & & & \\
$50,1,4,-$ & $4 /-2$ & $1 /-8$ & $88 / 88$ & $62 / 64$ & $73 / 76$ & $35 / 40$ \\
$50,3,4,-$ & $4 /-3$ & $2 /-7$ & $87 / 88$ & $61 / 65$ & $71 / 76$ & $26 / 41$ \\
& & & & & & \\
$50,2,1,-$ & $4 /-2$ & $4 /-6$ & $83 / 84$ & $57 / 60$ & $59 / 65$ & $1 / 19$ \\
$50,2,8,-$ & $4 /-3$ & $1 /-8$ & $88 / 88$ & $63 / 65$ & $74 / 76$ & $35 / 42$ \\
$50,2,16,-$ & $5 /-5$ & $1 /-11$ & $88 / 87$ & $62 / 65$ & $73 / 73$ & $36 / 39$ \\
& & & & & & \\
$50,2,4,1 \mathrm{ln}$ & $-/ 2$ & $-/ 0$ & $-/ 88$ & $-/ 65$ & $-/ 75$ & $-/ 40$ \\
$50,2,4, \gamma^{\prime}$ & $-/-3$ & $-/-8$ & $-/ 88$ & $-/ 65$ & $-/ 76$ & $-/ 42$ \\
$50,2,4, \mathrm{Wi}$ & $9 /-5$ & $0 /-10$ & $87 / 88$ & $65 / 70$ & $65 / 74$ & $40 / 48$ \\
$50,2,4, \mathrm{Su}$ & $2 /-1$ & $2 /-6$ & $87 / 88$ & $60 / 61$ & $73 / 76$ & $25 / 36$ \\
\hline
\end{tabular}




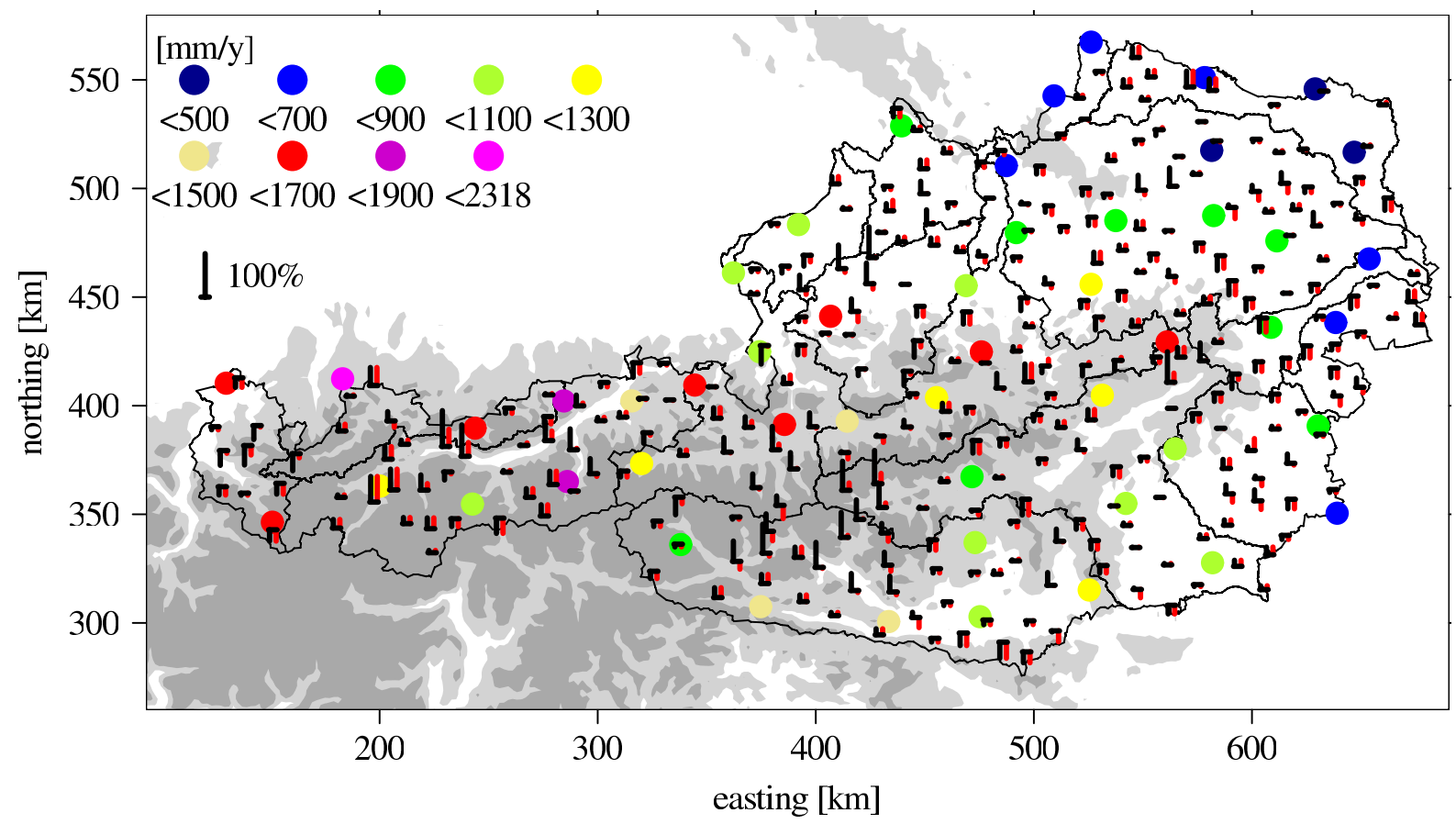

Fig. 8. As Fig. 7 but showing precipitation sums for the year 1999 at 50 observation sites and relative biases at the evaluation sites. The vertical bars indicate relative biases for the 1999 interpolation experiments. The inlet shows the height of a bar with $100 \%$ positive bias. Black gives biases with geographical and red with statistical distance next-neighbor interpolation.

spatial averages of these biases are given in Table 2 by experiment 50/2/1/- to 4 and -2 , respectively. Obviously, relative biases in $d$-IDW interpolation are larger in mountainous terrain than in the lowlands (the same is valid for correlation and efficiency errors, not shown). In mountainous terrain the scatter in relative biases is smaller with $\gamma$ - instead of $d$ interpolation and thus performance of $\gamma$-interpolation is better. This can also be seen in Fig. 9. This figure shows interpolated precipitation sums in comparison with observed sums of 1999 in a west-east transection with areal support of 350 to $370 \mathrm{~km}$ northing. Results with next- and four-neighbors interpolation are compared. Again the tendencies of over- and underestimation of geographical or statistical distance interpolation are visible. The tendency of smaller values of statistical interpolation yields smaller standard deviations. Subjectively interpolation with $n=4$ leads to better results, but obviously also to smoother, variability vastly underestimating fields.

The smaller scatter in bias, correlation and efficiency by $\gamma$-IDW is also shown by the histograms in Fig. 10. These histograms give statistics values applying $n=4$ interpolation. The statistical distance interpolation is more robust than geographical distance interpolation. Extreme overestimates of daily means are avoided. Correlations are shifted to higher values and the number of days with spatial $R^{2} \leq 0.5$ and small or even useless $(E \leq 0)$ efficiencies are significantly reduced.

Obviously, time series performance is better than spatial performance. This is due to the scales of the data. The temporal support of the data is daily. The spatial support of the observations $\left(\sim 1000 \mathrm{~cm}^{2}\right)$ is very small in comparison (Orlanski, 1975). This explains the better performance of interpolation in terms of time series than of spatial field comparisons. As a consequence the spatial results are more sensitive to the chosen interpolation method.

Most interpolation methods are smoothing operations which reduce field variability. This is visualized in the Taylor (2001)-diagram shown in Fig. 11. For example, nextneighbor $d$-interpolation overestimates temporal and spatial variability in comparison with the evaluating observations. But, with $n=2,4$ etc. variability is more and more underestimated. This effect is more important for spatial than temporal variability because of the relatively smaller spatial interpolation support scale. The traces of crosses in the diagram are convex showing that there is an optimum number of neighbors to be considered in interpolation. If in the envisaged application the field correlation is more important than field variability then a number of four neighbors is well chosen in case of 50 observing stations. The optimum depends on the interpolation setup: number of stations, power of distance, spatial and temporal variability of the natural precipitation field etc. The impact on smoothing of the power $\lambda$ of the distance and de-skewing in $\gamma$-IDW are also shown in Fig. 11. With increasing $\lambda$ the effective number of observations decrease and variability of the interpolating values 

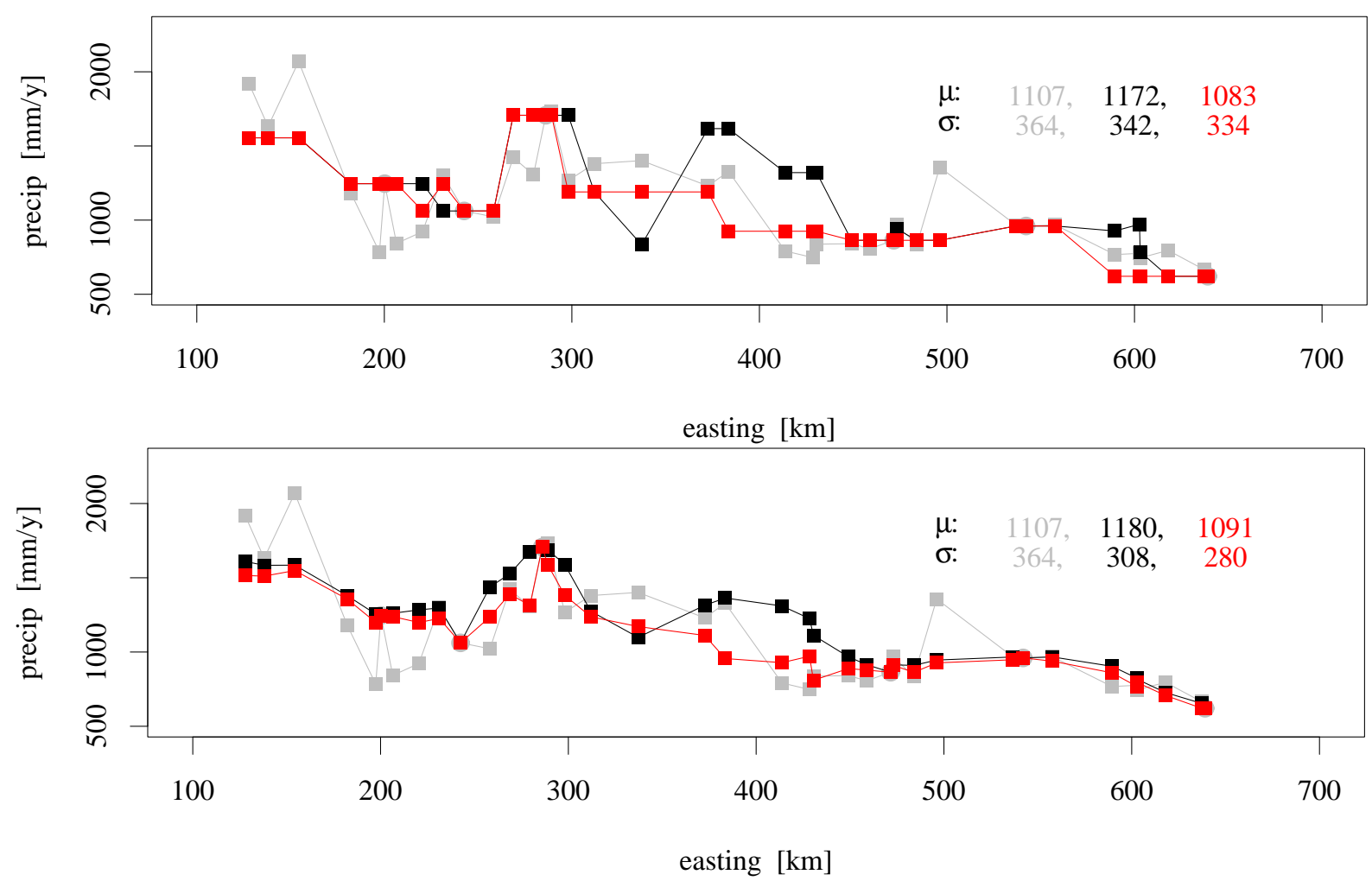

Fig. 9. Year 1999 sums of observed and interpolated precipitation at sites in a west-east transection with areal support between 350 and $370 \mathrm{~km}$ northing. Grey symbols show the observed values, black symbols the interpolated values with geographical distance interpolation and red symbols with statistical distance interpolation respectively. The upper panel applies $n=1$, i.e. next-neighbor, and the lower panel applies $n=4$ neighbors in interpolation. The transection means $\mu$ and standard deviations $\sigma$ are given too.

increase. De-skewing in $\gamma$ estimation slightly improves variability, correlation and thus (as is proven in Taylor, 2001) centered root-mean-square error RMSE'.

As discussed earlier there are systematic differences between station time series due to vertical or horizontal trends. In the interpolation experiments on a daily data basis these systematic effects are generally small in comparison to interpolation errors as is shown by application of $\gamma^{\prime}$ instead of $\gamma$ distances (cf. experiment $\gamma^{\prime}$ in Table 2). This says that on average the relative importance of vertical dependence of precipitation rates is small in comparison with interpolation errors in our setup. Of course, in some areas or applications the vertical dependence is important. Additionally, these systematic effects get more important with increasing interpolation performance, for example because of increasing temporal support of interpolation time slices (i.e. monthly or yearly precipitation fields instead of daily fields).

Seasonal stratification of precipitation events in $\gamma$ estimation and -interpolation yields the expected results. Table 2 gives interpolation results if only Summer or Winter six-month data are considered (experiments $\mathrm{Su}$ and Wi). Spatial field correlations and efficiencies are better in Winter than in Summer. In Austria the Winter precipitation is less intensive and less heterogeneous in the mean than Summer precipitation. The more stochastic character of convective Summer rain reduces the spatial representativeness of data. Nonetheless, the temporal correlation differences are small.

Interestingly, the temporal efficiencies are even better in Summer than in Winter. The MSEs of the interpolated time series are smaller in Winter $\left(d: 7.6(\mathrm{~mm} / \mathrm{d})^{2}\right.$ and $\gamma$ : $\left.5.9(\mathrm{~mm} / \mathrm{d})^{2}\right)$ than in Summer $\left(d: 17.9(\mathrm{~mm} / \mathrm{d})^{2}\right.$ and $\gamma$ : $\left.15.9(\mathrm{~mm} / \mathrm{d})^{2}\right)$. But, if the MSEs are normalized with observed time series mean variance $\left(22\right.$ and $66(\mathrm{~mm} / \mathrm{d})^{2}$ in Winter and Summer, respectively) then Summer interpolation performs better than Winter interpolation in terms of time series comparison. In either case, Summer or Winter, $\gamma$-IDW performs better than $d$-IDW with performance gain more pronounced in Winter because of higher stationarity of spatial patterns caused by frontal interaction with orography.

Stratification of precipitation days with a mean wind direction classification for the lower atmospheric levels in the Eastern Alps (Steinacker, 1990) has been tried also. This even slightly reduces overall performance in $\gamma$ interpolation. The small scale $\gamma$-distances are not highly dependent on large scale wind direction. Additionally, the size of specific wind direction classes is small even in the available thirty year data sets and thus estimation of stratified $\gamma \mathrm{s}$ is not robust. 

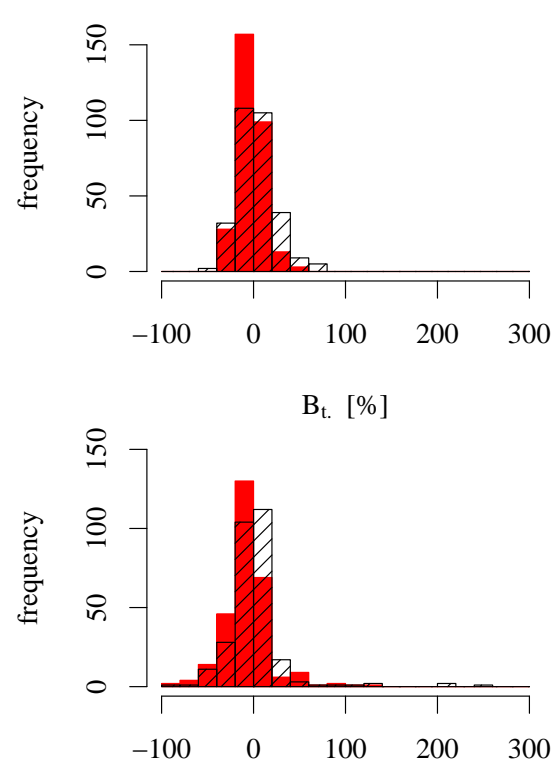

B.s $[\%]$

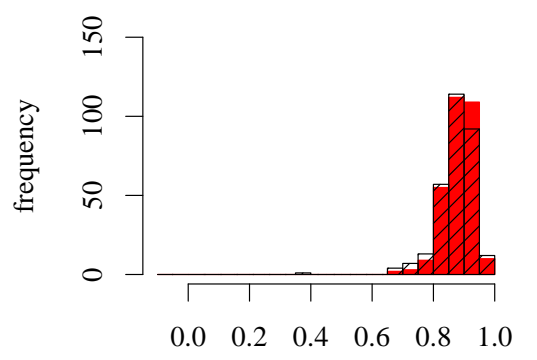

$\mathrm{R}_{\mathrm{t} .}$

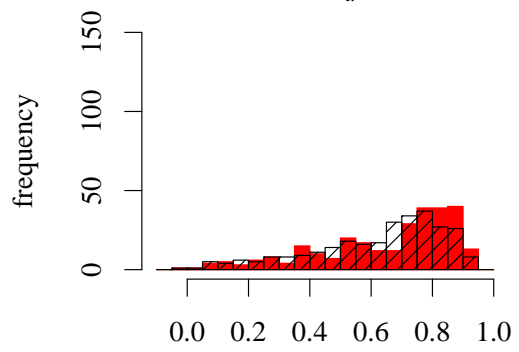

$\mathrm{R} . \mathrm{s}$

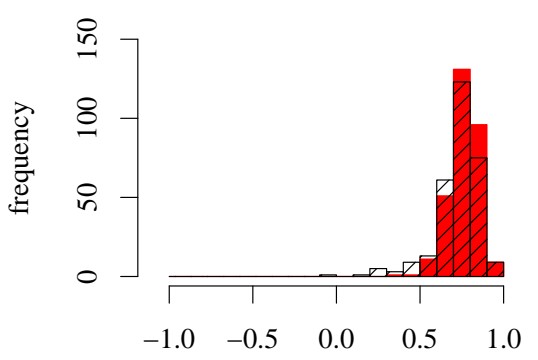

$\mathrm{E}_{\mathrm{t}}$

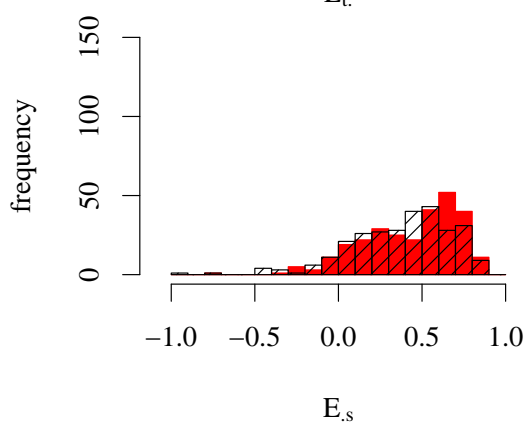

Fig. 10. Histograms of spatially (upper row) and temporally (lower row) averaged statistics. The solid red histograms show the evaluation results for $\gamma$-and the hatched black histograms for the $d$-distance interpolation with set 50, $n=4$, and $\gamma=2$.

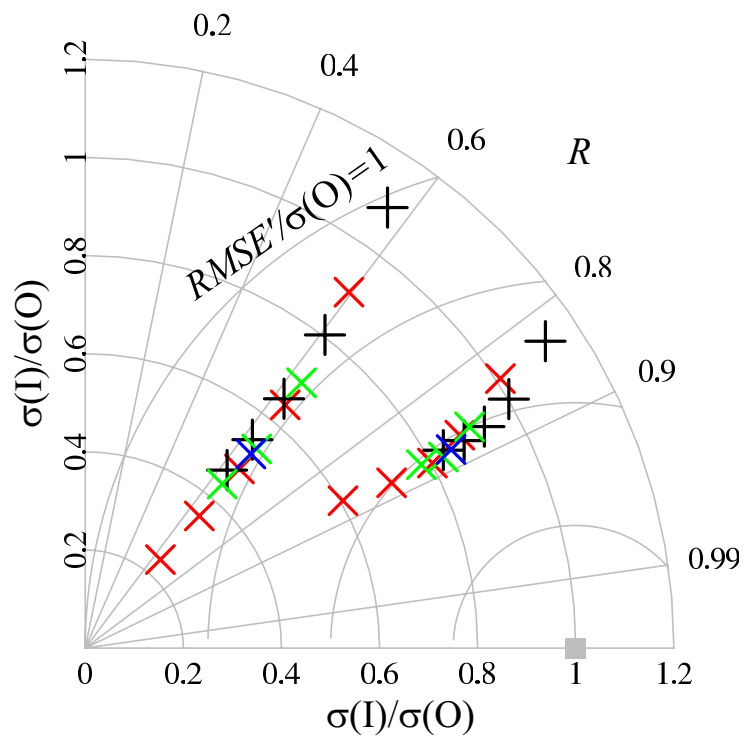

Fig. 11. Taylor (2001)-diagram showing evaluation results with observation set 50 but different interpolation setups. Black "+" and red "x" show the dependency on $n$ with $n=1,2,4,8,16$ for $d$ - and $\gamma$-interpolation, respectively, and $\gamma=2$. The green " $\mathrm{x}$ " show results with $\gamma$-interpolation and $n=4$ and $\gamma=1,3,4$. The blue "x" show results for experiment $l n$. The group of symbols indicating better correlations and centered RMSE's are from time series comparisons and the other group indicate spatial field comparisons.

\section{Conclusions}

Spatial interpolation of daily rain gauge data with Inverse Distance Weighting (IDW) at locations with available precipitation time series has been investigated. It has been shown that the application of a statistical distance measure between neighbored precipitation time series instead of geographical distances between station locations slightly improves averaged interpolation performance. The main advantage is that statistical distance IDW is more robust especially in or close to mountainous terrain where complex rain-orography interaction is important that is implicitly considered in the statistical distance. This performance gain in mountainous terrain illustrates the potential of simple but necessarily spatially highly resolving models of rain-orography interaction.

Geostatistical interpolation methods consider statistical relationship between stations through variogram or correlation models. Often, geostatistical models are implemented locally, i.e. they consider $n$ next neighbors in interpolation. These $n$ neighbors could easily be selected by explicit use of statistical distance. More sophisticated approaches could be thought of. For example, Kriging could be applied after mapping station locations with statistical distances instead of geographical distances by metric multidimensional scaling (Sammon, 1969). Thus, standard Kriging could be applied in statistical space, but this is a topic for further research.

Implementation of IDW interpolation with statistical distance is easily done if the necessary time series are available at the interpolation sites. An example of application might be 
daily precipitation mapping in Austria. Operationally about 150 rain gauges with daily or better resolution are available to the Austrian national weather service. Following Weilguni (2003) about 950 additional rain gauges with daily measurements are operated in Austria by the hydrological service, hydropower agencies etc. These additional stations are not available in near real-time, but their statistical information could be applied easily within the statistical IDW. This would be a parsimonious and robust procedure for using all available rain gauge data in densification of the point data network that could be appropriately upscaled in precipitation mapping.

Acknowledgements. Data are provided by the Hydrographische Zentralbüro, BMLFUW, Vienna. The author is funded by the Austrian Academy of Sciences.

Edited by: L. G. Lanza

\section{References}

Adler, R. F., Kidd, C., Petty, G., Morissey, M., and Goodman, H. M.: Intercomparison of Global Precipitation Products: The Third Precipitation Intercomparison Project (PIP-3), Bull. Amer. Meteorol. Soc., 82, 1377-1396, 2001.

Auer, I., Böhm, R., Jurković, A., Orlik, A., Potzmann, R., Schöner, W., Ungersböck, M., Brunetti, M., Nanni, T., Magueri, M., Briffa, K., Jones, P., Efthymiadis, D., Mestre, O., Moisselin, J.M., Begert, M., Brazdil, R., Bochnicék, O., Cegnar, T., GajićCapka, M., Zaninović, K., Majstorović, C., Szalai, S., Szentimrey, T., and Mercalli, L.: A new instrumental precipitation dataset for the greater Alpine region for the period 1800-2002, Int. J. Climatol., 25, 139-166, doi:10.1002/joc.1135, 2005.

Barros, A. P. and Lettenmaier, D. P.: Dynamic Modeling of orographically induced precipitation, Rev. Geophys., 32, 265-284, 1994.

Beck, A., Ahrens, B., and Stadlbacher, K.: Impact of nesting strategies on precipitation forecasting in dynamical downscaling of reanalysis data, Geophys. Res. Lett., 31, 5, doi:10.1029/2004GL020115, 2004.

Blöschl, G. and Grayson, R.: Spatial Observations and interpolation, in: Spatial patterns in catchment hydrology: observations and modelling, edited by: Grayson, R. and Blöschl, G., Cambridge University Press, UK, ISBN 0-521-63316-8, 17-50, 2001.

Ciach, G., Morrissey, M., and Krajewski, W. F.: Conditional bias in radar rainfall estimation, J. Appl. Meteorol., 39, 1941-1946, 2000.

Creutin, J. D. and Obled, C.: Objective analyses and mapping techniques for rainfall fields: An objective comparison, Water Resour. Res., 18, 413-431, 1982.

Daly, C., Neilson, R., and Phillips, D.: A statistical-topographic model for mapping climatological precipitation over mountainous terrain, J. Appl. Meteorol., 33, 140-158, 1994.

Durand, Y., Brun, E., Mérindol, L., Guyomarc'h, G., Lesafre, B., and Martin, E.: A meteorological estimation of relevant parameters for snow schemes used with atmospheric models, Ann. Glaciol., 18, 65-71, 1993.
Eischeid, J. K., Pasteris, P. A., Diaz, H. F., Plantico, M. S., and Lott, N. J.: Creating a serially complete, national daily time series of temperature and precipitation for the Western United States, J. Appl. Meteorol., 39, 1580-1591, 2000.

Frei, C. and Schär, C.: A precipitation climatology of the Alps from high-resolution rain-gauge observations, Int. J. Climatol., 18, 873-900, 1998.

Haiden, T. and Stadlbacher, K.: Quantitative Prognose des Fächenniederschlags, Österr. Wasser- und Abfallwirtschaft, 10, 135-141, 2002.

Liebmann, B. and Allured, D.: Daily precipitation grids for South America, Bull. Amer. Meteorol. Soc., 86, 1567-1570, doi:10.1175/BAMS-86-11-1567, 2005.

Orlanski, I.: A rational subdivision of scales for atmospheric processes, Bull. Amer. Meteorol. Soc., 56, 527-530, 1975.

Palecki, M. A., Angel, J. R., and Hollinger, S. E.: Storm precipitation in the United States. Part I: Meteorological characteristics, J. Appl. Meteorol., 44, 933-946, doi:10.1175/JAM2243.1, 2005.

Rubel, F.: PIDCAP Quick look precipitation atlas, vol. 15 of Österr. Beitr. Meteorol. Geophys., ZAMG, Wien, 1996.

Rubel, F. and Hantel, M.: BALTEX 1/6-degree daily precipitation climatology 1996-1998, Meteorol. Atmos. Phys., 77, 155-166, 2001.

Rudolf, B. and Rubel, F.: Global precipitation, in: Observed Global Climate, edited by: M. Hantel, Landolt-Börnstein: Numerical Data and Functional Relationships in Science and Technology New Series, Group 5: Geophysics, 6(A), 11.1-11.53, Springer, Berlin, 2005.

Sammon Jr., J.: A nonlinear mapping for data structure analysis, IEEE Trans. Comput., C-18, 401-409, 1969.

Scheifinger, H., Böhm, R., and Auer, I.: Räumliche Dekorrelation von Klimazeitreihen unterschiedlicher zeitlicher Auflösung und ihre Bedeutung für ihre Homogenisierbarkeit und die Repräsentativität von Ergebnissen, in Proceedings of 6. Deutsche Klimatagung, Klimavariabilität 2003, 22-25 September 2003, Potsdam, no. 6 in Terra Nostra, Schriftenreihe der AlfredWegener-Stiftung, pp. 375-379, 2003.

Sevruk, B.: Regional dependency of precipitation-altitude relationship in the Swiss Alps, Climatic Change, 36, 355-369, 1997.

Shepard, D.: Computer mapping: The SYMAP interpolation algorithm, in: Spatial statistics and models, edited by: Gaile, G. and Willmott, C., Dordrecht, Reidel Publishing, p. 95-116, 1984.

Singh, V. and Frevert, D. (Eds.): Mathematical Models of Large Watershed Hydrology, Water resources Publications, LLC, Chelsea, Michigan, 2002a.

Singh, V. and Frevert, D. (Eds.): Mathematical Models of Small Watershed Hydrology and Applications, Water resources Publications, LLC, Chelsea, Michigan, 2002b.

Skoda, G., Weilguni, V., and Haiden, T.: Heavy convective storms - Precipitation during 15, 60, and 180 minutes, chap. 2.5-7, Hydrological Atlas of Austria, Bundesministerium für Land- und Forstwirtschaft, Umwelt und Wasserwirtschaft, 2003.

Smith, R.: The influence of mountains on the atmosphere, Adv. Geophys., 21, 87-230, 1979.

Smith, R.: A linear upslope-time-delay model for orographic precipitation, J. Hydrol., 282, 2-9, 2003.

Steinacker, R.: Eine ostalpine Strömungslagenklassifikation, Tech. rep., IMG; Universität Wien, Austria, http://www.univie.ac.at/ IMG-Wien/weatherregime/, pp. 8, 1990. 
Taylor, K.: Summarizing multiple aspects of model performance in a single diagram, J. Geophys. Res., 106, 7183-7192, 2001.

Tobler, W.: A computer movie simulating urban growth in the Detroit region, Economic Geography, 46, 234-240, 1970.

Weilguni, V.: Precipitation stations, chap. 2.1, Hydrological Atlas of Austria, Bundesministerium für Land- und Forstwirtschaft, Umwelt und Wasserwirtschaft, 2003.
Young, C., Nelson, B., Bradley, A., Smith, J., Peters-Lidard, C., Kruger, A., and Baeck, M.: An evaluation of NEXRAD precipitation estimates in complex terrain, J. Geophys. Res., 104, 19691-19703, 1999. 